\title{
EPiT : A Software Testing Tool for Generation of Test Cases Automatically
}

\author{
Rosziati Ibrahim $^{\# 1}$, Ammar Aminuddin Bani Amin ${ }^{\# 2}$, Sapiee Jamel ${ }^{\# \# 3}$, Jahari Abdul Wahab ${ }^{* 4}$ \\ ${ }^{\#}$ Department of Software Engineering, Universiti Tun Hussein Onn Malaysia, 86400, Malaysia \\ ${ }^{\#}$ Department of Information Security,, Universiti Tun Hussein Onn Malaysia, 86400, Malaysia \\ *Engineering R\&D Department, Sena Traffic Systems Sdn. Bhd., Kuala Lumpur, 57000, Malaysia
}

\begin{abstract}
Software test cases can be defined as a set of condition where a tester needs to test and determine that the System Under Test (SUT) satisfied with the expected result correctly. This paper discusses the optimization technique in generating cases automatically by using EpiT (Eclipse Plug-in Tool). EpiT is developed to optimize the generation of test cases from source code in order to reduce time used for conventional manually creating test cases. By using code smell functionality, EpiT helps to generate test cases automatically from Java programs by checking its line of code (LOC). The implementation of EpiT will also be presented based on several case studies conducted to show the optimization of the test cases generated. Based on the results presented, EpiT is proven to solve the problem for software tester to generate test case manually and check the optimization from the source code using code smell technique.
\end{abstract}

Keywords - Software Testing, Test Cases, Code Smell, Source Code, System Optimization, Line of Code;

\section{INTRODUCTION}

There are many techniques for a software tester to conduct a SUT within testing phase. Typically, a software tester manually generates the test cases and testing each of the module. This technique however may take longer time where most of the activity of generating test cases are prone to redundancy [1]. Moreover, a software tester needs to be precisely validate the test cases well in order to avoid ambiguity presented in Software Test Plan documentation. In a nut shell, automation tool helps to increase the reliability of system while reducing the cost of manual software testing [2 - 4].

To solve the problem of manually generating test cases, EpiT can be used as a software testing tool where test cases can be automatically generated from the source code. By using the code smell functionality, the tool can generate test cases by examining line by line of the source codes. Thus, EpiT optimization will help to find and provide high quality solutions.

This paper discusses the optimization technique using code smell algorithm in order to reduce the generation of test cases due to redundancy of test cases as well as an approach to automate the process of generating the test cases. The rest of the paper is organized as follows. It consists of 7 Sections. Related works are discussed in Section 2 and Section 3 discuss the application involved. Meanwhile, Section 4 demonstrates the framework of EpiT. Section 5 provides the implementation of EpiT tool using code smell to generate test cases from source code. Section 6 shows the results after the implementation phase and conclusion is in Section 7.

\section{RELATED WORK}

Research paper of Albert [5] presents jPET which is a white-box test-case generator (TCG). jPET is a software testing tool build on Eclipse environment which automatically generate test-cases from the bytecode compiled from the java class. In order to yield this information to the user at the source code level, jPET performs reverse engineering of the testcases obtained at the bytecode level by PET. By using jPET as integrated Eclipse programming, software developers can directly conduct testing activities in development phase.

In the study by Zhenzhen Wang, and Qiaolian Liu [6] discuss an improved Particle Swarm Optimization (PSO) algorithm to generate test case automatically. This study evaluated several techniques such as PSO and Genetic algorithm to determine perfomance in term of generating test cases. Based on the result, shows that the improved PSO is effectively reduce run time in generating software test cases automatically compare to conventional PSO.

The study by Hanyu Pei et al. [7] proposed a cloud-based Dynamic Random Test (DRT) technique for handling the evaluation of test case prioritization and resource allocation. This study also evaluated several techniques between the cloud-DRT and other techniques such as Round Robin Schedule (RRS) and cloud-based Random Partition Testing (RPT). All these experiments of evaluation were conducted to find fault detection effectiveness. Based on the result, it was concluded that the proposed technique uses less test cases to detect all seeded faults compared to RRS and cloud-based RPT. AMOGA [8] is another study for software testing. AMOGA is used as a framework in generating test cases from GUI for mobile applications.

Yunqi Du et al. [9] proposed a hybrid techniques which merging a mutation testing technique with 
genetic algorithm. These combination technique will be able to design test case optimization methods. The method helps in producing the test cases generation. Based on the results, they are able to show their combination technique have better score in term of an average variation score of more than $95 \%$ on the variants of the complete set. Thus, this study proved that using mutation testing combine with genetic algorithm leads to higher coverage and mutation score within test cases.

Based on study by Annibale Panichella et al. [10] conducted on 346 Java classes to assess the performance of DynaMOSA compared with wholesuite approach (WS), its archive-based variant (WSA) and Many-Objective Sorting Algorithm (MOSA). By Using DynaMOSA technique, test case can be generated and maximize test coverage in SUT. Based on the experimental result, it shows that DynaMOSA improved 8 percent in average coverage compared to MOSA technique. Table 1 shows the summary of the related works discussed.

TABLE I: SUMMARY OF RELATED WORK

\begin{tabular}{|c|c|c|c|}
\hline No & $\begin{array}{c}\text { Authors \& } \\
\text { Year }\end{array}$ & Techniques & Outcome \\
\hline 1 & $\begin{array}{l}\text { Elvira Albert, } \\
\text { (2011) [5] }\end{array}$ & $\begin{array}{l}\text { jPET: an } \\
\text { Automatic Test- } \\
\text { Case Generator } \\
\text { Plugin }\end{array}$ & $\begin{array}{l}\text { Fully integrated } \\
\text { test case generation } \\
\text { within the software } \\
\text { development } \\
\text { process. }\end{array}$ \\
\hline 2 & $\begin{array}{l}\text { Zhenzhen } \\
\text { Wang, } \\
\text { Qiaolian Liu } \\
\text { (2018) [6] }\end{array}$ & $\begin{array}{l}\text { Particle Swarm } \\
\text { Optimization } \\
\text { (PSO) algorithm }\end{array}$ & $\begin{array}{l}\text { The technique } \\
\text { proved that is } \\
\text { better compared to } \\
\text { conventional PSO. }\end{array}$ \\
\hline 3 & $\begin{array}{l}\text { Hanyu Pei et } \\
\text { al. } \\
\text { (2018) [7] }\end{array}$ & $\begin{array}{l}\text { Dynamic } \\
\text { Random Testing } \\
\text { Strategy (DRT) }\end{array}$ & $\begin{array}{l}\text { The technique } \\
\text { proposed more } \\
\text { effective in } \\
\text { generating test } \\
\text { cases compared to } \\
\text { RPT and RRS. }\end{array}$ \\
\hline 4 & $\begin{array}{l}\text { Salihu et al. } \\
\text { (2018) [8] }\end{array}$ & AMOGA & $\begin{array}{l}\text { AMOGA is used as } \\
\text { a framework for } \\
\text { testing mobile } \\
\text { applications by } \\
\text { using GUI }\end{array}$ \\
\hline 5 & $\begin{array}{l}\text { Yunqi Du et } \\
\text { al. } \\
\text { (2019) [9] }\end{array}$ & $\begin{array}{l}\text { Hyrbid } \\
\text { Technique } \\
\text { merging } \\
\text { Mutation Testing } \\
\text { Techniques with } \\
\text { Genetic } \\
\text { Algorithm }\end{array}$ & $\begin{array}{l}\text { The hybrid } \\
\text { techniques leads to } \\
\text { better coverage and } \\
\text { better mutation } \\
\text { score in generating } \\
\text { test cases. }\end{array}$ \\
\hline 6 & $\begin{array}{l}\text { Annibale } \\
\text { Panichella et } \\
\text { al. } \\
(2018)[10]\end{array}$ & $\begin{array}{l}\text { Dynamic Many- } \\
\text { Objective } \\
\text { Sorting } \\
\text { Algorithm } \\
\text { (DynaMOSA), }\end{array}$ & $\begin{array}{l}\text { The technique } \\
\text { proved to more } \\
\text { high coverage in } \\
\text { test case generation } \\
\text { compared with } \\
\text { MOSA. }\end{array}$ \\
\hline
\end{tabular}

\section{EPIT FRAMEWORK}

In this section, the framework of EpiT is clearly defined to demonstrate how the generation of test cases are derived. Fig. 1 shows the framework for EPiT. Source code is the input for EPiT. Then, the parser will be used to read line by line of the source codes. EPiT will detect classes with code smell and then generate test cases automatically based on detected methods.

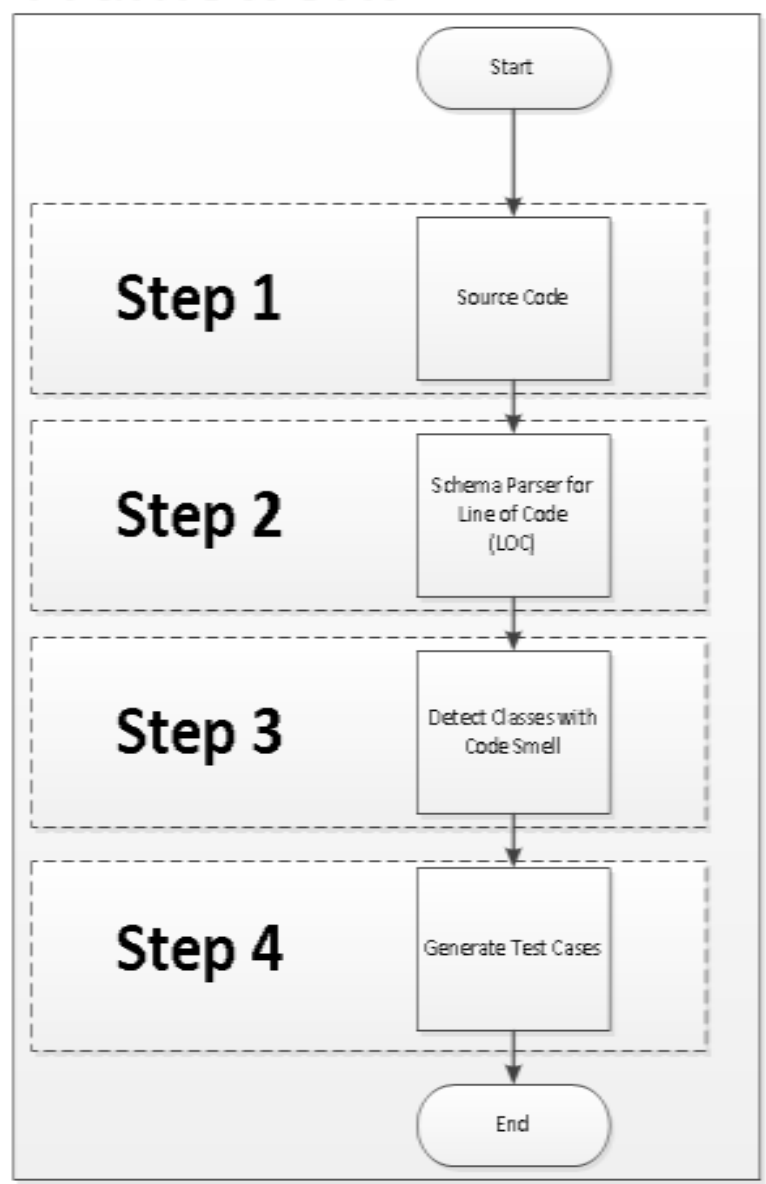

Fig. 1: The Framework of EPiT

Based from Fig. 1, a software tester can generate the test cases using EpiT in Eclipse Integrated Development Environment (IDE). Eclipse IDE is always been used to develop wide scope of application because of open source license. However, the development of EpiT for source code is currently limited to Java programming only. The details discussion for the framework has been discussed in [11].

\section{THE CASE STUDIES}

In this paper, several applications were implemented in order to test the Epit framework. All four of the applications were selected and downloaded from open source community Github.

The first application is the calendar application [12] which are used to view yearly and monthly calendar given input from user. Additionally, this 
application also calculates age where the application count the duration from birth of date to current year.

Secondly, the BlackJack application [13] is a card game where each player will dealt a card until a certain point. Player who get the highest hand win the round. The application consists of seven classes in total.

Thirdly, the CoffeeMaker application [14] is an inventory system for making a coffee. A user can add, edit or delete each coffee recipe to the inventory. Moreover, user can view all the listed recipes in the inventory. This application consists of four classes in total.

Lastly, the Elevator application [15] is an elevator system where allocate elevator cart to assign floor. Many functions were embed in the application such as the elevator cart will repositioned itself to the lowest floor when in idle state. This application also check sequence queue and sorted which floor should be stop first.

\section{IMPLEMENTATION OF EPIT TOOL}

Base from the framework of EPiT in Fig. 1, the tool is implemented according to four steps. These steps are discussed in this section.

\section{A. Step 1: Source Code}

Example of calendar case study using source code is shown in Fig. 2. The application consists of two methods in Java programming. Firstly, the project will be imported in Eclipse environment. Then the codes will be analyzed by EpiT by using the menu as shown in Fig. 3.

\section{B. Step 2: Schema Parser}

By default, EpiT will read line by line according to the algorithm. Epit uses Java parser to get an Abstract Syntax Tree (AST). Based on Fig. 4, example of the AST code where is a structure representing Java programming in a way that it is easy to check and validate.

\section{Detect Classes with Code Smell}

Epit will start to analyze and detect all the classes in the source using code smell functionality [16]. The parser will detect the class by node. Then within each of the node, EpiT identifies as method which has several other attributes such as method name, return type and input parameter. All the method classes detected will be stored in variable in EpiT. Fig. 5 shows example of code smell functionality in EpiT.

\section{Generate Test Cases}

Then, test cases are generated based on the attributes that have been identify within the code smell functionality. Each of the test cases will have at least three possible scenario such as valid input, invalid input and null input. Example is shown in Fig. 6 where each of the test cases will be generated with multiple scenario.

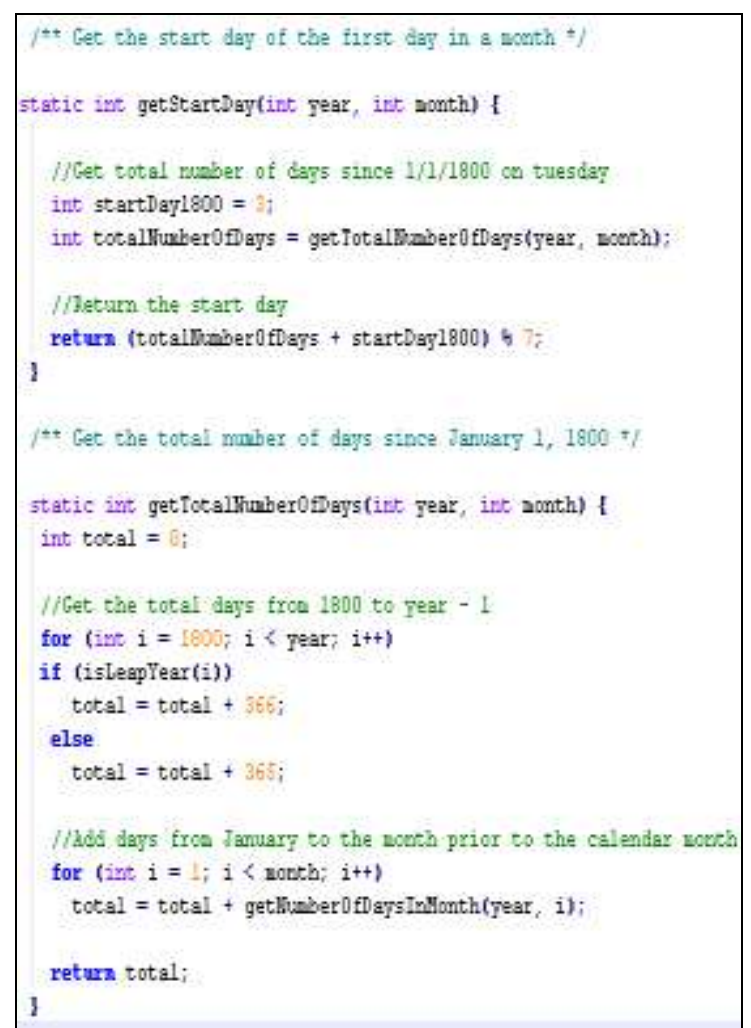

Fig. 2: Source Codes for Calender

\begin{tabular}{l} 
Iu uc ma \\
Validate \\
Restore from Local History... \\
Web Services \\
EPiT Pro- Analyze Code \\
Team \\
Compare With \\
Replace With \\
\hline Properties
\end{tabular}

Fig. 3: EPiT Analyzer

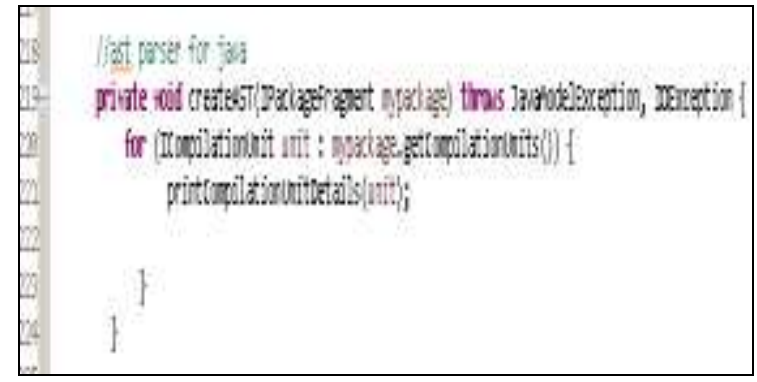

Fig. 4: AST Code

After EPiT has finished analyzed all the source code, it will prompt the success popup. Fig. 7 shows example of the popup with time duration taken on each process. 


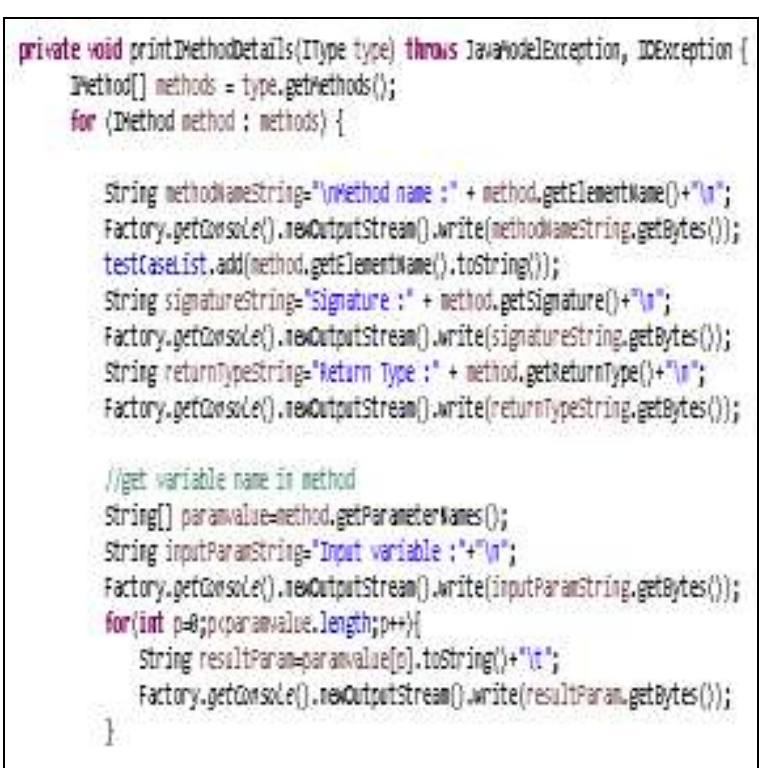

Fig. 5: Code Smell in EPiT

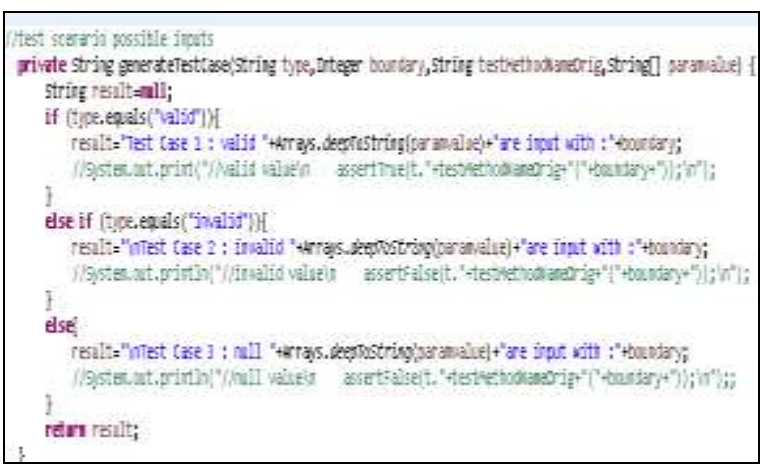

Fig. 6: Generation of Test Cases

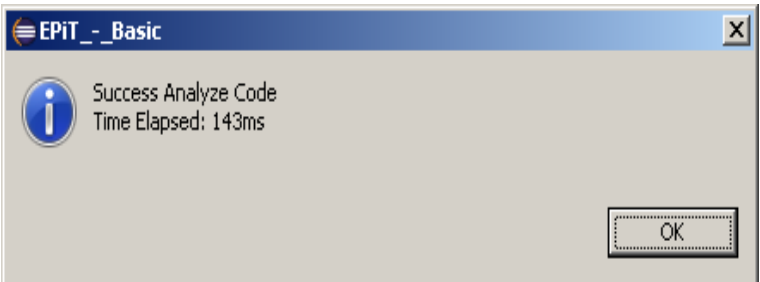

Fig. 7: Success Dialog Box in EPiT

Next, during analyzing the source codes, EPiT will output all details in the EpiT console. The console will show many attributes such as time and date of the conducted process, name of the project, and details each of test cases generated. Fig. 8 shows that the EpiT console of each of analyzed process.

Finally, EPiT will summarize all the analyzed source code and generated test cases in the EpiT console. The console will show project name, total generated test cases, duration of each process conducted. Fig. 9 shows that the EpiT summarization of each of successful process.

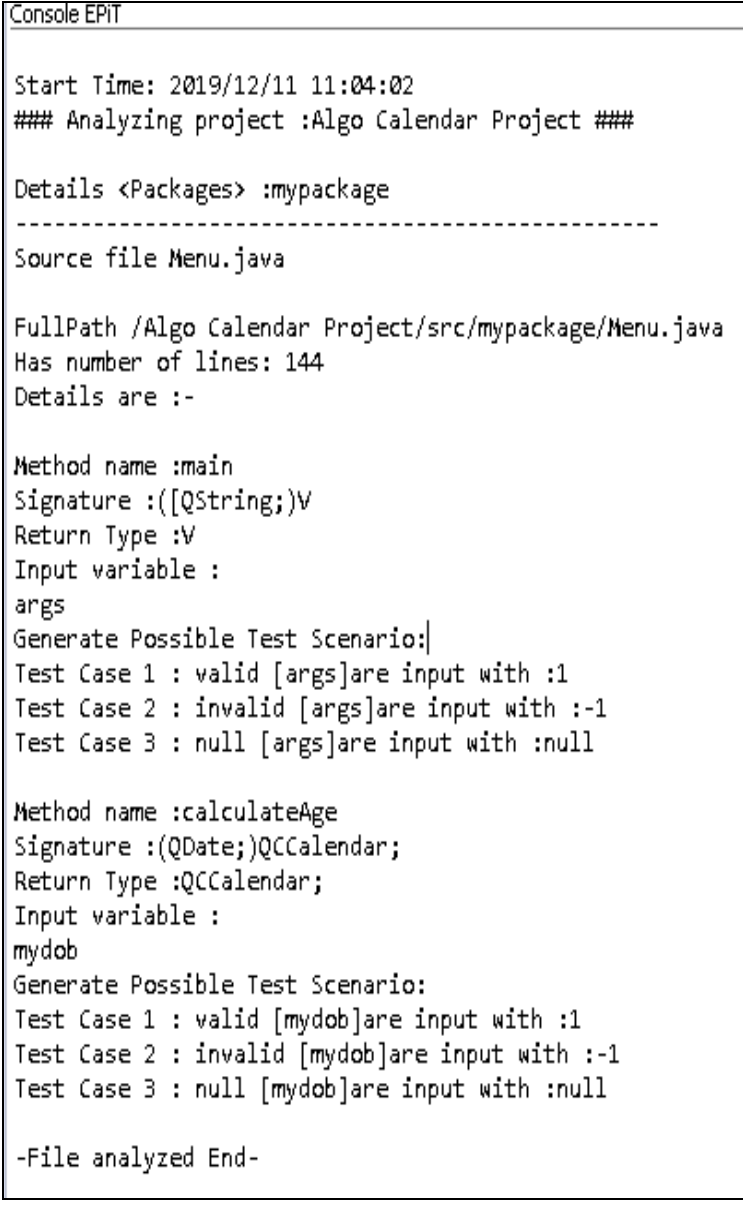

Fig. 8: The Console for EPiT

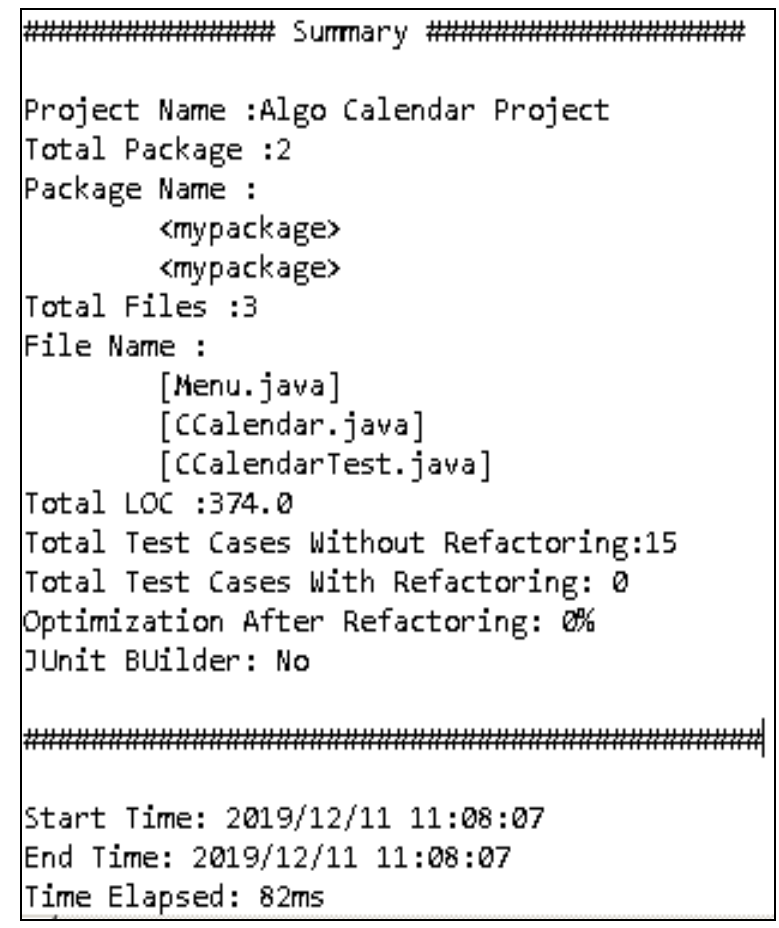

Fig. 9: Summary of EPiT Analyzer 


\section{RESULTS AND DISCUSSIONS}

Based on Table II, comparison result between each of case study is shown. The result shows that the calendar application is the fastest with $82 \mathrm{~ms}$ and generated fifteen test cases. Meanwhile, the slowest is the coffee maker application which is $206 \mathrm{~ms}$ with fifty seven test cases were generated.

The comparison had been led by considering one perspective. The differences of each application by duration time to generate test cases. From fastest time which is $81 \mathrm{~ms}$ and the slowest time which is $206 \mathrm{~ms}$.

There are many factors which lead to inconsistent result which are project code complexity, CPU usage and memory usage. Although the result is not consistent, this project had proven that EpiT is faster than conventional manually generated test cases. Thus, those studies support the result of this project.

TABLE II: RESUlTS OF CASE STUdIES

\begin{tabular}{|l|l|l|l|}
\hline Application & \multicolumn{1}{|c|}{$\begin{array}{c}\text { Total } \\
\text { LOC }\end{array}$} & $\begin{array}{l}\text { Test Cases } \\
\text { Generated }\end{array}$ & $\begin{array}{c}\text { Duration } \\
\text { Time }(\mathrm{ms})\end{array}$ \\
\hline Calendar & 374 & 15 & 82 \\
\hline BlackJack & 1017 & 41 & 163 \\
\hline CoffeeMaker & 982 & 57 & 206 \\
\hline Elevator & 1183 & 66 & 149 \\
\hline
\end{tabular}

\section{CONCLUSIONS}

This paper has discussed the works of the generating test cases using EpiT in Eclipse environment. Through the experiment, this research verified that EpiT is more reliable and faster response time compared to conventional manual testing technique. By analyzing the result, test cases can be optimized and help software tester to remove any redundancy in the source code. Based on the results, it is proven that using code smell functionality is a very efficient way to automatically generate test cases from source code. Other techniques for software testing can also be investigated and implemented such as mutation software testing technique, regression software testing technique and fuzzy software testing technique.

\section{ACKNOWLEDGMENT}

The authors would like to thanks Ministry of Education (MOE) for supporting this study under Prototype Research Grant (PRGS) Vote No K037.

\section{REFERENCES}

[1] Ibaraki, S., Tsujimoto, S., Nagai, Y., Sakai, Y, Morimoto, S., Miyazaki, Y. (2018). "A pyramid-shaped machining test to identify rotary axis error motions on five-axis machine tools: software development and a case study", International Journal of Advanced Manufacturing Technology, 94(1-4): 227-237, DOI: 10.1007/s00170-017-0906-9

[2] Janczarek, P. and Sosnowski, J. (2015). "Investigating software testing and maintenance reports: Case study", Information and Software Technology, 58: 272-288, DOI: 10.1016/j.infsof.2014.06.015

[3] Chen, J., Zhu, J., Chen, T.Y., Towey, D., Kuo, F.C., Huang, R., Guo, Y. (2018). "Test case prioritization for objectoriented software: An adaptive random sequence approach based on clustering", Journal of Systems and Software, 135: 107-125, DOI: 10.1016/j.jss.2017.09.031

[4] Chen, T.-H.,Thomas, S.W.,Hemmati, H., Nagappan, M., Hassan, A.E. (2017). "An Empirical Study on the Effect of Testing on Code Quality Using Topic Models: A Case Study on Software Development Systems", IEEE Transactions on Reliability, 66(3): 806-824, DOI: 10.1109/TR.2017.2699938

[5] Albert, E. (2011). "jPET: an Automatic Test-Case Generator for Java". 18th Working Conference on Reverse Engineering, 441-442.

[6] Wang,Z., Liu,Q. (2018). "A Software Test Case Automatic Generation Technology Based on the Modified Particle Swarm Optimization Algorithm". International Conference on Virtual Reality and Intelligent Systems (ICVRIS). Retrieved November 20, 2019, from: https://ieeexplore.ieee.org/document/8531372

[7] Pei,H., Yin,B., Xie, M. (2018). "Dynamic Random Testing Strategy for Test Case Optimization in Cloud Environment." IEEE International Symposium on Software Reliability Engineering Workshops (ISSREW). Retrieved November 20, 2019, from: https://ieeexplore.ieee.org/document/8539185

[8] Salihu, I.A., Ibrahim, R., Ahmed, B.S., Zamli, K.Z. Usman, A. (2019). "AMOGA: A Static-Dynamic Model Generation Strategy for Mobile Apps Testing”. IEEE Access. 2019. DOI:10.1109/ACCESS.2019.2895504

[9] Du,Y., Ao, H., Fan, Y., Pan, Y., O.Alex. (2019). "Automatic Test Case Generation and Optimization Based on Mutation Testing." IEEE 19th International Conference on Software Quality, Reliability and Security Companion (QRS-C). Retrieved December 1, 2019, from: https://ieeexplore.ieee.org/document/8859495

[10] Panichella, A., Kifetew, F. M., and Tonella, P. (2018). "Automated Test Case Generation as a Many-Objective Optimization Problem with Dynamic Selection of the Target". IEEE Transactions on Software Engineering, Volume: 44, Issue: 2, Feb. 1 2018. Retrieved December 1, 2019, from: https://ieeexplore.ieee.org/document/7840029

[11] Ibrahim, R., Ahmed, M., Jamel, S. (2019). "An Eclipse Plugin Tool for Generating Test Cases from Source Codes". Proceedings of the 2019 Asia Pacific Information Technology Conference. DOI: 10.1145/3314527.3314535

[12] Xechnologi, (2019).“Calendar Project.” Retrieved December 1, 2019, from: https://github.com/xechnologi/my-repo

[13] Brown,J., (2015). BlackJack. Retrieved December 1, 2019, from: https://github.com/jbbrown93/BlackJack

[14] Behrens, A. (2014). "Coffee Maker". Retrieved December 1, 2019, from: https://github.com/serious6/CoffeMaker

[15] Strang, T., Bauer, C. (2017). Elevator Scheduling Simulator. Retrieved December 1, 2019 from: https://github.com/00111000/Elevator-Scheduling-Simulator

[16] Ibrahim, R., Ahmed, M., Nayak, R., Jamel, S. (2018). "Reducing redundancy of test cases generation using code smell detection and refactoring". Journal of King Saud University. DOI: 10.1016/j.jksuci.2018.06.005 